\title{
Slicings of parallelogram polyominoes, or how Baxter and Schröder can be reconciled
}

\author{
Mathilde Bouvel ${ }^{1}$, Veronica Guerrini $\Downarrow$, and Simone Rinaldi ${ }^{2}$ \\ ${ }^{1}$ Institut für Mathematik, Universität Zürich, Switzerland. \\ ${ }^{2}$ Dipartimento di Ingegneria dell'Informazione e Scienze Matematiche, Università degli Studi di Siena, Italy.
}

\begin{abstract}
We provide a new succession rule (i.e. generating tree) associated with Schröder numbers, that interpolates between the known succession rules for Catalan and Baxter numbers. We define Schröder and Baxter generalizations of parallelogram polyominoes (called slicings) which grow according to these succession rules. We also exhibit Schröder subclasses of Baxter classes, namely a Schröder subset of triples of non-intersecting lattice paths, and a new Schröder subset of Baxter permutations.

Résumé. Nous décrivons une nouvelle règle de réécriture (c'est-à-dire un nouvel arbre de génération) correspondant aux nombres de Schröder, qui s'intercale entre les règles de réécriture connues pour les nombres de Catalan et de Baxter. Nous définissons des généralisations des polyominos parallélogrammes comptées par les nombres de Schröder et de Baxter dont la croissance est régie par ces règles de réécriture. Nous illustrons aussi notre travail en donnant des sous-classes de Schröder dans des classes de Baxter, en particulier un sous-ensemble de triplets de chemins nonintersectant énuméré par les nombres de Schröder, et une nouvelle sous-famille de Schröder dans l'ensemble des permutations de Baxter.
\end{abstract}

Keywords. Parallelogram polyominoes, Generating trees, Baxter numbers, Schröder numbers, Catalan numbers, Non-intersecting lattice paths

Some proofs are sketched or omitted in this extended abstract. Details may be found in [5].

\section{Introduction}

The sequence of Catalan numbers (A000108 in [14]) is arguably the most well-known combinatorial sequence. It is known to enumerate dozens of families of combinatorial objects, among which Dyck paths, parallelogram polyominoes, or $\tau$-avoiding permutation ${ }^{(i)}$ for any permutation $\tau$ of size 3 . In this paper, we are interested in Catalan numbers as well as in two larger combinatorial sequences: the Schröder and Baxter numbers.

Baxter numbers (sequence A001181 in [14]) were first introduced in [9], where it is shown that they count Baxter permutations. They also enumerate numerous families of combinatorial objects, and their

\footnotetext{
${ }^{\dagger}$ Corresponding author. Email: guerrini55estudent.unisi.it

(i) Recall that a permutation $\sigma=\sigma_{1} \sigma_{2} \ldots \sigma_{n}$ contains $\tau=\tau_{1} \tau_{2} \ldots \tau_{k}$ if there exists $i_{1}<i_{2}<\ldots<i_{k}$ such that $\sigma_{i_{a}}<\sigma_{i_{b}}$ if and only if $\tau_{a}<\tau_{b}$. Otherwise $\sigma$ avoids $\tau$.
}

1365-8050 @ 2016 Discrete Mathematics and Theoretical Computer Science (DMTCS), Nancy, France 
study has attracted significant attention, see for instance [3, 10]. Many such Baxter families can be immediately seen to contain a Catalan subfamily. For instance, the set of triples of non-intersecting lattice paths (NILPs from here on) contains all pairs of NILPs (that are in essence parallelogram polyominoes, see Figure 1); and Baxter permutations, defined by the avoidance of the vincular (ii) patterns 2413 and $3 \underline{14} 2$, include $\tau$-avoiding permutations, for any $\tau \in\{132,213,231,312\}$.

On the other hand, the (large) Schröder numbers (sequence A006318) seem to be a bit less popular. They also form a sequence point wise larger than the Catalan sequence, and it is additionally point wise smaller than the Baxter sequence. This transpires easily on permutations, where the Schröder numbers are counting the separable permutations [13, 15], defined by the avoidance of 2413 and 3142.

The main purpose of this article is to explain and illustrate the inclusions "Catalan in Schröder in Baxter". Although these inclusions are obvious on pattern-avoiding permutations, they remain quite obscure on other objects. Indeed, looking at several combinatorial objects, it appears that the permutation example is a little miracle, and that the unclarity of these inclusions is rather the rule here. To give only a few examples, consider for instance lattice paths: the Dyck paths generalize into Schröder paths (by allowing an additional flat step of length 2), but have to our knowledge no natural Baxter analogue; on the contrary, pairs of NILPs are counted by Catalan, whereas triples of NILPs are counted by Baxter, leaving Schröder aside. Or, consider another well-known Catalan family: that of binary trees. There are Schröder and Baxter objects generalizing binary trees (like Schröder trees, with an additional sign on the root on one hand, or pairs of twin binary trees on the other), but they have apparently nothing in common.

As these examples illustrate, the Baxter and Schröder generalizations of Catalan objects are often independent and are not easily reconciled. This fact is also visible at a more abstract level, i.e. without referring to specific combinatorial families: by considering the generating trees (with their corresponding succession rules) associated with these sequences (we will review the basics of generating trees in Section 2). As we demonstrate in this work, for the known generating trees associated with the Schröder and Baxter numbers, when they can be seen as generalizations of the generating tree of Catalan numbers, then these two generalizations go in two opposite directions. Our main contribution is to provide a continuum from Catalan to Baxter via Schröder, that is visible at the abstract level of succession rules. Specializing these on particular objects, it allows us to define compatible Schröder and Baxter generalizations of Catalan objects. We will focus mostly on generalizations of parallelogram polyominoes, that we call slicings of parallelogram polyominoes. Section 3 defines our Baxter slicings (showing also their tight connection with triples of NILPs). These new objects allow us to see that the usual Baxter succession rule does nothing but symmetrize the Catalan succession rule. In Section 4 , we introduce a new succession rule associated with Schröder numbers, that interpolates between the Catalan and Baxter rules of Sections 2 and 3 Letting our slicings grow with this rule allows us to define the family of Schröder slicings. From there, the last two sections go in different directions. Section 5 presents other Schröder subclasses of Baxter classes, obtained via our new Schröder succession rule: in the case of triples of NILPs and of permutations. In Section 6, we introduce more intermediate classes between Catalan and Baxter, refining our new Schröder succession rule with a parameter that may vary.

(ii) Note that we do not represent vincular patterns with dashes, as it was done originally. We prefer the more modern and more coherent notation that indicates by a symbol $\_$the elements of the pattern that are required to be adjacent in an occurrence. The definition of avoidance of a vincular pattern will be reviewed in Section 3 


\section{Parallelogram polyominoes and the Catalan generating tree}

There are many ways of defining (or characterizing) parallelogram polyominoes in the literature, and we only give one that fits our needs.

Definition 1 A parallelogram polyomino $P$ (see an example on Figure 1 a $)$ ) is an (edge-)connected set of unit cells in the Cartesian plane, that is the interior of a contour defined by two paths, which are composed of $(0,1)$ and $(1,0)$ steps and which never meet except at their beginning and end. Denoting $(k, \ell)$ the dimension of the minimal bounding rectangle of $P$, the semi-perimeter of $P$ is $k+\ell$, and the size of $P$ is $k+\ell-1$.

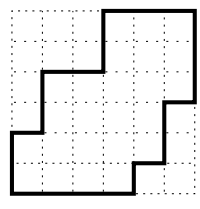

(a)

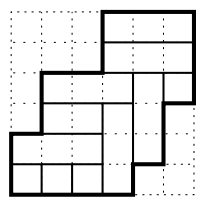

(b)

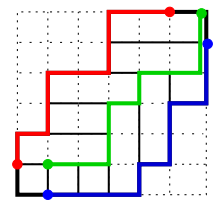

(c)

Fig. 1: (a) A parallelogram polyomino $P$ of size 11, (b) a Baxter slicing of shape $P$, and (c) the triple of NILPs associated with it.

We start by reviewing generating trees [1, 2, 15], and in particular the generating tree for Catalan numbers associated with parallelogram polyominoes.

A generating tree for a combinatorial class $\mathcal{C}$ is a infinite rooted tree, whose vertices are the objects of $\mathcal{C}$, each appearing exactly once in the tree, and such that objects of size $n$ are at distance $n$ from the root (with the convention that the root is at distance 1 from itself, and is labeled by the only object of size 1 in $\mathcal{C})$. The children of some object $c \in \mathcal{C}$ are obtained adding an atom (i.e., a piece of object that makes its size increase by 1) to $c$. Clearly, every object should appear only once in the tree, so not all additions are possible. We must ensure the unique appearance property by considering only additions that follow some restricted rules. We call the growth of $\mathcal{C}$ the process of adding atoms following these prescribed rules.

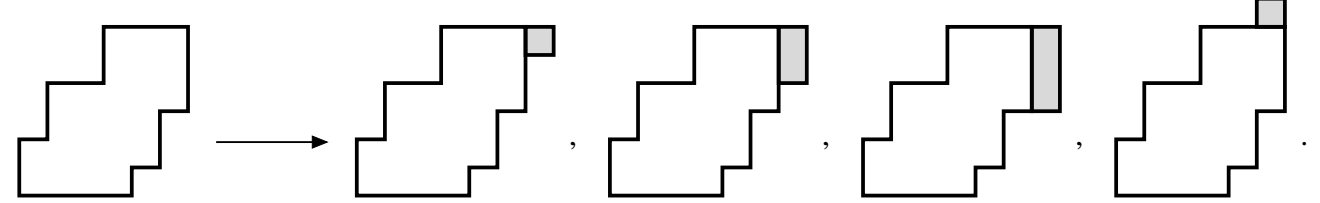

Fig. 2: The growth of parallelogram polyominoes.

A generating tree of parallelogram polyominoes was described in [2], and the corresponding growth is illustrated on Figure 2 The atoms that may be inserted are rightmost columns (of any possible height from 1 to the height of the current rightmost column), and topmost rows of width 1 . Note that the restriction on the width of the new row added is here only to ensure that no polyomino is produced several times. Note also that the symmetric growth, that allows rows of any admissible width but columns of height 1 only, also describes a generating tree for parallelogram polyominoes, which is isomorphic to the first one.

All that matters to us is the shape of a generating tree, forgetting the combinatorial objects on the vertices. In what follows, we will use the phrase "generating tree" to denote this shape only, referring instead to "full generating trees" when the nodes are carrying combinatorial objects. 
Generating trees become substantially useful if they can be described in an abstract way, without referring to the details of the combinatorial objects. More precisely, for a combinatorial class $\mathcal{C}$, assuming that there is a statistics on the objects of $\mathcal{C}$, whose value determines the number of children in the full generating tree, then the (shape of the) generating tree depends only on how the value of the statistics evolves from a object to its children. When such a statistics exists, we give labels to the objects of $\mathcal{C}$, which indicate the value of the statistics. The associated succession rule is then given by the label of the root and, for any label $k$, the labels of the children of an object labeled by $k$. A succession rule characterizes completely a generating tree.

In the case of parallelogram polyominoes, the number of children is determined by the height of the rightmost column (namely, it is this height +1 ), and it is easy to follow the height of the rightmost column along their growth. It follows that the generating tree of parallelogram polyominoes described above is completely determined by the following succession rule:

$$
\text { root labeled }(1) \quad \text { and } \quad(k) \rightsquigarrow(1),(2), \ldots,(k),(k+1) .
$$

We will denote this generating tree by $\mathcal{T}_{\text {Cat }}$.

Note that, given a succession rule and its subsequent generating tree, we can associate with it an enumeration sequence, whose $n$-th term $c_{n}$ is the number of vertices in the tree at distance $n$ from the root. Of course, $\left(c_{n}\right)$ is the enumeration sequence of any combinatorial class that has a (full) generating tree encoded by the given succession rule. But our point, which will be essential later on, is that the sequence may also be associated directly with the generating tree, without reference to any combinatorial class. On our example, it follows that rule (Cat) (and the corresponding tree $\mathcal{T}_{\text {Cat }}$ ) is associated with the Catalan numbers, hence its name.

\section{Baxter slicings}

\subsection{A Baxter succession rule generalizing Catalan}

There are several succession rules associated with Baxter numbers [4, 6, 7, 8]. We will be interested in one of these rules only which, in addition to being the most well-known, is the one that generalizes the rule for Catalan number in the most natural way. The rule is:

$$
\text { root labeled }(1,1) \quad \text { and } \quad(h, k) \rightsquigarrow\left\{\begin{array}{l}
(1, k+1),(2, k+1), \ldots,(h, k+1), \\
(h+1,1),(h+1,2), \ldots,(h+1, k) .
\end{array}\right.
$$

We denote by $\mathcal{T}_{\mathrm{Bax}}$ the generating tree associated with this rule. A proof that it corresponds to Baxter numbers can be found in [4, 11], where it is proved that the Baxter permutations grow according to rule $(\mathrm{Bax})$. Recall that Baxter permutations are those avoiding the vincular patterns $2 \underline{41} 3$ and $3 \underline{14} 2$, i.e. permutations $\sigma$ such that no subsequence $\sigma_{i} \sigma_{j} \sigma_{j+1} \sigma_{k}$ satisfies $\sigma_{j+1}<\sigma_{i}<\sigma_{k}<\sigma_{j}$ or $\sigma_{j}<\sigma_{k}<$ $\sigma_{i}<\sigma_{j+1}$. From [4, 11], the growth of Baxter permutations according to rule (Bax) consists, for any Baxter permutation $\sigma$, in inserting a new maximum element either immediately to the left of a left-toright maximum of $\sigma$, or immediately to the right of a right-to-left maximum of $\sigma$. The label $(h, k)$ of a permutation records the number of its left-to-right maxima (for $h$ ) and right-to-left maxima (for $k$ ).

It is easily seen, however rarely noticed, that rule $\overline{\mathrm{Bax}}$ generalizes rule (Cat) (so that $\mathcal{T}_{\text {Bax }}$ contains a subtree isomorphic to $\left.\mathcal{T}_{\text {Cat }}\right)$. Indeed, the production of label $(h, k)$ in rule $(\overline{B a x})$ includes labels $(h+1, i)$ 
for $1 \leq i \leq k$ and label $(1, k+1)$, for instance ${ }^{(\text {iiii) }}$ Keeping track of the second element of the label only gives back the Catalan rule (Cat). Moreover, for another subset of the labels produced, the same holds keeping track of the first element only.

In some sense, rule $\overline{\mathrm{Bax}}$ is just the symmetric version of rule (Cat). This is very well understood on the growth of parallelogram polyominoes according to rule Cat. As we have seen, with rule (Cat), a rightmost column may be added, of all possible heights; but only a topmost row of width 1 is allowed. But the symmetric variant of this rule, allowing addition of a topmost row of all possible widths, and of a rightmost column of height 1 , also works. So we can think of rule $(\mathrm{Bax})$ as generating parallelogram polyominoes symmetrically, allowing at the same time the insertion of a rightmost column of any possible height, or of a topmost row of any possible width. Of course, this process generates the parallelogram polyominoes ambiguously.

\subsection{Definition and growth of Baxter slicings}

Our remark that rule $\overline{\mathrm{Bax}}$ generates parallelogram polyominoes symmetrically but ambiguously motivates the definition of new combinatorial objects, that generalize parallelogram polyominoes, and grow unambiguously according to rule $(\overline{B a x})$. From the discussion above, the natural generalization is to let parallelogram polyominoes grow according to rule $\overline{\mathrm{Bax}}]$ as we explain, but to record the "building history" of the polyomino, that is, which columns and rows where added by the growth process. The objects obtained are parallelogram polyominoes whose interior is divided into blocks, of width or height 1 . We call these objects Baxter slicings of parallelogram polyominoes, or Baxter slicings for short.

Definition 2 A Baxter slicing (see an example on Figure 1 b)) of size $n$ is a parallelogram polyomino $P$ of size $n$ whose interior is divided into $n$ blocks as follows: one block is the topmost row (resp. rightmost column) of $P$ - such blocks are called horizontal (resp. vertical) blocks -, and the other $n-1$ blocks form a Baxter slicing of the parallelogram polyomino of size $n-1$ obtained by deletion of the topmost row (resp. rightmost column) of $P$.

Theorem 3 Baxter slicings grow according to rule $(\mathrm{Bax})$ and are enumerated by Baxter numbers.

Proof: It is clear that Baxter slicings grow according to rule $(\mathrm{Bax})$ : a Baxter slicing has label $(h, k)$ when the topmost row has width $h$ and the rightmost column has height $k$, and the productions of label $(h, k)$ are immediately seen to correspond to the Baxter slicings obtained adding a new horizontal block in a new topmost row, of any width between 1 and $h$, or a new vertical block in a new rightmost column, of any height between 1 and $k$. As a consequence, Baxter slicings are enumerated by Baxter numbers.

\subsection{Bijection with NILPS}

Among the combinatorial families enumerated by Baxter numbers, one can be seen to be in bijection with Baxter slicings in a very simple way, namely, the triples of NILPs.

Definition 4 A path of size $n$ is a sequence of $\operatorname{North}(N=(0,1))$ and East $(E=(1,0))$ steps, containing $n-1$ steps in total. Given three paths $u, m$, and $d$ of the same size $n$, all containing the same number of $E$ (and $N$ ) steps, $(u, m, d)$ is a triple of non-intersecting lattice paths (for short, triple of NILPs) of size

(iii) Remark that, comparing the growth of Baxter slicings (defined later) with that of parallelogram polyominoes, it is natural to consider label $(1, k+1)$. But choosing $(h, k+1)$ instead would of course also satisfies our purpose. 
$n$ when the embeddings of $u, m$ and $d$ in the plane never meet, with $u$ (resp. $m$, resp. $d$ ) starting at the point of coordinates $(0,2)$ (resp. $(1,1)$, resp. $(2,0)$ ).

Theorem 5 The following construction, illustrated on Figure 1 c) provides a size-preserving bijection between Baxter slicings and triples of NILPs:

Consider a Baxter slicing of a parallelogram polyomino $P$, whose bottom-left corner is assumed to be placed at coordinates $(0,0)$. Define the paths

- u corresponding to the upper border of $P$, except the first and last steps,

- $d$ corresponding to the lower border of $P$, except the first and last steps,

- and $m$ going from $(1,1)$ to the top-right corner of $P$, following the lower border of every

horizontal block of the slicing, and the right border of every vertical block, and associate the triple $(u, m, d)$ to the original Baxter slicing.

Up to the simple bijective correspondence described in Theorem 5, our Theorem 3 can also be seen as a description of the growth of triples of NILPs according to the generating tree $\mathcal{T}_{\mathrm{Bax}}$, which was already described in [3].

\section{Schröder slicings}

Our interest in defining Baxter slicings is to find a family of objects enumerated by the Schröder numbers, that lie between parallelogram polyominoes and Baxter slicings, and which grow according to a succession rule that generalizes (Cat) while specializing $\overline{\mathrm{Bax}}$. Note that to our knowledge, out of the many succession rules for Schröder numbers [12, 15], none has this property.

\subsection{A new Schröder succession rule}

Let us consider the following succession rule, whose associated generating tree is denoted $\mathcal{T}_{\text {Sch }}$ :

$$
\text { root labeled }(1,1) \quad \text { and } \quad(h, k) \rightsquigarrow\left\{\begin{array}{l}
(1, k+1),(2, k+1), \ldots,(h, k+1), \\
(2,1),(2,2), \ldots,(2, k-1),(h+1, k) .
\end{array}\right.
$$

Theorem 6 The enumeration sequence associated with rule (NewSch is that of Schröder numbers.

Proof: From [15], we know that the following succession rule is associated with Schröder numbers:

$$
\text { root labeled }(2) \quad \text { and } \quad(j) \rightsquigarrow(3),(4), \ldots,(j),(j+1),(j+1) .
$$

We claim that rules $(\mathrm{NewSch}$ and $(\mathrm{Sch})$ produce the same generating tree. Indeed, replacing each label $(h, k)$ in rule (NewSch) by the sum $h+k$ of its elements immediately gives rule $\mathrm{Sch}$.

It is not obvious that rule $(\mathrm{Sch})$ generalizes rule $(\mathrm{Cat})$, ensuring that $\mathcal{T}_{\text {Sch }}$ contains a subtree isomorphic to $\mathcal{T}_{\text {Cat }}$. But this becomes clear with rule (NewSch), which can be immediately seen to generalize rule (Cat), in the same fashion rule $(\mathrm{Bax})$ does. Indeed, in rule (NewSch, looking only at the productions $(2,1),(2,2), \ldots,(2, k-1),(h+1, k)$ and $(1, k+1)$ of a label $(h, k)$, and considering the second component of the labels, we recover rule (Cat).

What is further interesting with rule $(\mathrm{NewSch})$ is that rule $(\overline{\mathrm{Bax}})$ for Baxter numbers generalizes it. Indeed, the only difference between them is that labels $(h+1, i)$ for $1 \leq i \leq k-1$ in the production of 
rule $\overline{\mathrm{Bax}}$ are replaced by $(2, i)$ in rule (NewSch). From this remark, we easily show by induction on the depth reached in the generating trees that the generating tree produced from root $(h, k)$ in rule (NewSch) is (isomorphic to) a subtree of the generating tree produced from root $\left(h^{\prime}, k\right)$ in rule $(\overline{\mathrm{Bax}})$, for all $h^{\prime} \geq h$. All together this proves our claim that $\mathcal{T}_{\text {Sch }}$ is (isomorphic to) a subtree of $\mathcal{T}_{\text {Bax }}$. Moreover, along the proof, we exhibit a subtree of $\mathcal{T}_{\text {Bax }}$ isomorphic to $\mathcal{T}_{\text {Sch }}$ (obtained by mapping the productions in rules $\mathrm{Bax}$ and (NewSch) in the obvious way), which we call "canonical".

To our knowledge, this is the first time three succession rules for Catalan, Schröder and Baxter numbers are given, which are each a generalization of the previous one.

\subsection{Definition of Schröder slicings, and their growth}

We want to define Schröder slicings so that they form a subset of the Baxter slicings, that is enumerated by the Schröder numbers, and whose growth is described by rule (NewSch). To do that, we can use the "canonical" subtree of $\mathcal{T}_{\text {Bax }}$ isomorphic to $\mathcal{T}_{\text {Sch }}$ mentioned earlier: it is enough to label the vertices of $\mathcal{T}_{\text {Bax }}$ by the corresponding Baxter slicings, and to keep only the objects which label a vertex of this canonical subtree. With this global approach to the definition of Schröder slicings, the problem is to provide a characterization of these objects that would be local, i.e. that could be checked on any given Baxter slicing without reconstructing the whole chain of productions according to rule $(\mathrm{Bax})$ that resulted in this object.

For the sake of clarity, we have chosen to reverse the order in the presentation of Schröder slicings, that is to say, we will first give their "local characterization", and then prove (see Theorem 9 ) that they grow according to rule (NewSch). It is also not hard to prove from the explicit description of the "canonical" subtree of $\mathcal{T}_{\text {Bax }}$ isomorphic to $\mathcal{T}_{\text {Sch }}$ (ommited in this extended abstract) that Schröder slicings are those labeling the vertices of this subtree when $\mathcal{T}_{\text {Bax }}$ is considered on Baxter slicings.

Definition 7 Let $B$ be a Baxter slicing of a parallelogram polyomino $P$, and $u$ be a horizontal block of $B$. We denote by $\ell(u)$ the width of $u$. The projection $X(u)$ of $u$ on the lower border of $P$ is the lower-most point of this border whose abscissa is that of the right edge of $u$. We now define $r(u)$ to be the number of horizontal steps on the lower border of $P$ to the left of $X(u)$ before a vertical step (or the bottom-left corner of $P$ ) is met.

Definition 8 A Schröder slicing is any Baxter slicing such that for any horizontal block $u$, the following inequality holds: $\ell(u) \leq r(u)+1$. We denote this condition ( $\left.\ell r_{1}\right)$.

Figure 3 a,b) illustrates the definitions of $\ell(u)$ and $r(u)$, and shows an example of Schröder slicing.

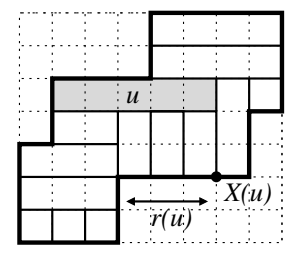

(a)

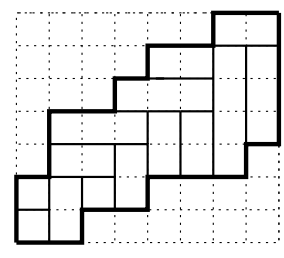

(b)

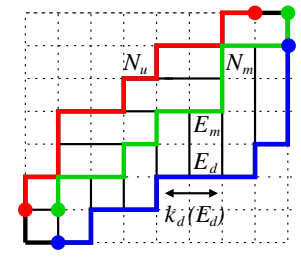

(c)

Fig. 3: (a) Illustration of Definition 7 (b) example of Schröder slicing, and (c) illustration of Definition 10

Theorem 9 A generating tree for Schröder slicings is $\mathcal{T}_{\text {Sch }}$, associated with rule (NewSch). 
Proof: Like Baxter slicings, Schröder slicings grow adding vertical blocks on the right and horizontal blocks on top, but whose width is restricted, so that condition $\left(\ell r_{1}\right)$ is always satisfied.

To any Schröder slicing $P$, let us associate the label $(h, k)$ where $h$ (resp. $k$ ) denotes the maximal width (resp. height) of a horizontal (resp. vertical) block that may be added to $P$, without violating condition $\left(\ell r_{1}\right)$. Note that if a horizontal block of width $i$ may be added, then for all $i^{\prime} \leq i$, the addition of a horizontal block of width $i^{\prime}$ is also allowed. Consequently, we may add horizontal blocks of width 1 to $h$ to $P$. Moreover, $k$ denotes the height of the rightmost column of $P$ (since condition $\left(\ell r_{1}\right)$ introduces no restriction on vertical blocks), and columns of any height from 1 to $k$ may be added to $P$.

Figure 4 illustrates the growth of Schröder slicings according to rule (NewSch). From a Schröder slicing of label $(h, k)$, we may add a new horizontal block of width $i \leq h$ (resp. a new vertical block of height $k$, resp. a new vertical block of height $j<k$ ), which gives a Schröder slicing of label $(i, k+1)$ (resp. $(h+1, k)$, resp. $(2, j))$. Details are provided in [5].

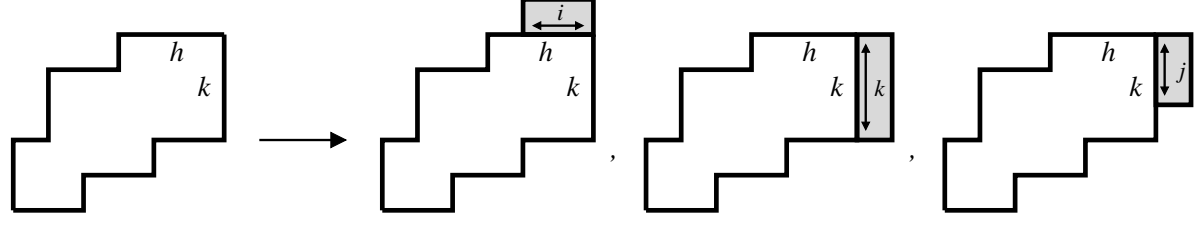

Fig. 4: The productions of a Schröder slicing of label $(h, k)$ following rule (NewSch).

\section{Other Schröder restrictions of Baxter objects}

For any Baxter class $\mathcal{C}$, whose growth according to rule $(\overline{\mathrm{Bax}})$ is understood, it is immediate to define a Schröder subclass of $\mathcal{C}$. Indeed, we can consider the full generating tree of shape $\mathcal{T}_{\text {Bax }}$ associated with $\mathcal{C}$, its "canonical" subtree isomorphic to $\mathcal{T}_{\text {sch }}$ discussed earlier, and keep only the objects of $\mathcal{C}$ associated with a vertex of $\mathcal{T}_{\text {Sch }}$. This method has the advantage of being systematic, but it does not provide a characterization of the objects in the Schröder subclass which does not refer to the generating trees.

In this section, we give two examples of Schröder subclasses of Baxter classes, that are not obtained with the above general method, but for which we provide a characterization of the Schröder objects without reference to generating trees.

\subsection{A Schröder family of NILPS}

From Theorem 5, we have a simple bijection between triples of NILPs and Baxter slicings. And in Section 4 , we have seen a subset of Baxter slicings enumerated by the Schröder numbers. A natural question, that we now solve, is then to give a characterization of the triples of NILPs which correspond to Schröder slicings via the bijection of Theorem 5

Definition 10 Let $(u, m, d)$ be a triple of NILPs as in Definition 4.

A pair $\left(N_{u}, N_{m}\right)$ of $N$ steps of $u$ and $m$ is matched if there exists $i$ such that $N_{u}$ (resp. $N_{m}$ ) is the $i$-th $N$ step of $u$ (resp. $m$ ). Similarly, a pair $\left(E_{m}, E_{d}\right)$ of $E$ steps of $m$ and $d$ is matched if there exists $i$ such that $E_{m}$ (resp. $\left.E_{d}\right)$ is the $i$-th E step of $m$ (resp. $d$ ).

Moreover, for any $N$ step $N_{u}$ in u (resp. $N_{m}$ in $\left.m\right)$, we denote by $h_{u}\left(N_{u}\right)\left(\right.$ resp. $\left.h_{m}\left(N_{m}\right)\right)$ the number of $E$ steps of $u$ (resp. $m$ ) that occur before $N_{u}$ (resp. $N_{m}$ ). And for any $E$ step $E_{d}$ in $d$, we denote by $k_{d}\left(E_{d}\right)$ the largest $k$ such that $E^{k}$ is a factor of d ending in $E_{d}$. 
See Figure 3 c) for an illustration of this definition. As suggested by this figure, it holds that a matched pair of $N$ steps of $u$ and $m$ (resp. of $E$ steps of $m$ and $d$ ) corresponds to the left and right (resp. top and bottom) edges of a horizontal (resp. vertical) block of the Baxter slicing associated with $(u, m, d)$.

Definition 11 A Schröder triple of NILPs is any triple $(u, m, d)$ as in Definition 4 such that for any $N$ step $N_{u}$ of the path $u$, denoting $N_{m}$ the $N$ step of $m$ such that $\left(N_{u}, N_{m}\right)$ is matched, $E_{m}$ the last $E$ step of $m$ before $N_{m}$, and $E_{d}$ the $E$ step of $d$ such that $\left(E_{m}, E_{d}\right)$ is matched, the following inequality holds:

$$
h_{u}\left(N_{u}\right)-h_{m}\left(N_{m}\right) \leq k_{d}\left(E_{d}\right) .
$$

Theorem 12 Schröder slicings are in one-to-one correspondence with Schröder triples of NILPS by means of the size-preserving bijection described in Theorem 5 .

Proof (sketch): Consider a Baxter slicing $P$ and the corresponding triple of NILPs $(u, m, d)$. The core of the proof is to see that, for any steps $N_{u}, N_{m}, E_{m}, E_{d}$ as in Definition 11, denoting $w$ the horizontal block of $P$ whose left edge is $N_{u}$, we have $\ell(w)=h_{m}\left(N_{m}\right)+1-h_{u}\left(N_{u}\right)$ and $r(w)=k_{d}\left(E_{d}\right)$.

\subsection{Another Schröder subset of Baxter permutations}

The class Sep $=\operatorname{Av}(2413,3142)$ of separable permutations is well-known to be a subset of the set Bax of Baxter permutations enumerated by the Schröder numbers. A generating tree for Sep following rule $\mathrm{Sch}$ has been described in [15], but we were not able to explain the growth of separable permutations according to rule $(\mathrm{NewSch}$. However, restricting the growth of Baxter permutations according to rule $(\overline{B a x})$, we were able to describe a new subset of Baxter permutations, enumerated by the Schröder numbers, and whose growth is governed by rule (NewSch.

As explained at the beginning of this section, a Schröder subset of Baxter permutations can be obtained by considering the "canonical" embedding of $\mathcal{T}_{\text {Sch }}$ in $\mathcal{T}_{\text {Bax }}$. Doing so, the two Baxter permutations of size 5 that are not obtained are 13254 and 23154. Although this subset of Baxter permutations is easy to define from the generating tree perspective, we have not been able to characterize the permutations it contains without referring to the generating trees, which is somewhat unsatisfactory. On the other hand, the subset of Baxter permutations studied below is not as immediate to define from the generating trees themselves, but has a nice characterization in terms of forbidden patterns.

The definition (in a special case) of bivincular patterns is useful to define the subset of Baxter permutations we are considering: a permutation $\sigma$ avoids the pattern $41323^{+}$(resp. $42313^{+}$) when no subsequence $\sigma_{i} \sigma_{j} \sigma_{k} \sigma_{\ell} \sigma_{m}$ of $\sigma$ satisfies $\sigma_{j}<\sigma_{\ell}<\sigma_{k}$ (resp. $\sigma_{\ell}<\sigma_{j}<\sigma_{k}$ ), $\sigma_{m}=\sigma_{k}+1$, and $\sigma_{m}<\sigma_{i}$.

Theorem 13 Let $\mathcal{S}$ be the subset of Baxter permutations defined by avoidance of the (bi)vincular patterns $2413,3142,41323^{+}$and $42313^{+}$. The generating tree obtained letting permutations of $\mathcal{S}$ grow by insertion of a maximal element is $\mathcal{T}_{\text {Sch }}$, and consequently $\mathcal{S}$ is enumerated by the Schröder numbers.

Note that the two Baxter permutations of size 5 that are not in $\mathcal{S}$ are 51324 and 52314.

\section{More families of restricted slicings}

\subsection{Catalan slicings}

Similarly to the path followed to define Schröder slicings, we can consider the generating tree $\mathcal{T}_{\text {Bax }}$ of Baxter slicings, and its subtree isomorphic to $\mathcal{T}_{\text {Cat }}$ discussed in Subsection 3.1. to define "Catalan slicings" of 
parallelogram polyominoes. As expected, we find exactly one Catalan slicing $C$ for every parallelogram polyomino $P$, namely, the Baxter slicing of shape $P$ whose horizontal blocks all have width 1 . Alternatively, $C$ can be recursively described as follows: if the top row of $P$ contains just one cell, then this cell constitutes a horizontal block of $C$, and we proceed computing the Catalan slicing of $P$ minus this top row; otherwise, the rightmost column of $P$ constitutes a vertical block of $C$, and we proceed computing the Catalan slicing of $P$ minus this rightmost column.

\subsection{Skinny slicings}

We have seen in Definition 8 that Schröder slicings are defined by the condition $\ell(u) \leq r(u)+1$, for any horizontal block $u$. This condition $\left(\ell r_{1}\right)$ can be naturally generalized for any non-negative integer $m$ as follows: for any horizontal block $u, \ell(u) \leq r(u)+m$. We denote this condition $\left(\ell r_{m}\right)$.

Definition 14 An $m$-skinny slicing is a Baxter slicing such that for any horizontal block $u$, the inequality $\left(\ell r_{m}\right)$ holds.

Theorem 15 A generating tree for m-skinny slicings is described by the following succession rule:

$$
\text { root labeled }(1,1) \quad \text { and }(h, k) \rightsquigarrow\left\{\begin{array}{ll}
(1, k+1),(2, k+1), \ldots,(h, k+1), & \\
(h+1,1), \ldots,(h+1, k-1),(h+1, k), & \text { if } h<m, \\
(m+1,1), \ldots,(m+1, k-1),(h+1, k) . & \text { if } h \geq m .
\end{array}\left(\Omega_{m}\right)\right.
$$

Proof: The proof follows the exact same steps as the proof of Theorem 9, which corresponds to $m=1$. The only difference is that the maximal width of the horizontal block that may be added in the third case is $\max (h+1, m+1)$ instead of 2 .

Considering the case $m=0$, we obtain a family of Baxter slicings which is intermediate between Catalan slicings (for which $\ell(u)=1$, for all horizontal blocks $u$ ) and Schröder slicings (i.e. 1-skinny slicings). The first few terms of the enumeration sequence of 0 -skinny slicings are $1,2,6,21,80,322, \ldots$. We give below a functional equation characterizing the generating function of 0 -skinny slicings, therefore proving that this sequence is (up to the first term) the same as sequence A106228 in [14].

Theorem 16 The generating function of 0-skinny slicings satisfies $F(x)=\frac{x(F(x)+1)}{1-x(F(x)+1)^{2}}$.

Proof (sketch): We apply the method of [1, 4]. We translate rule $\left(\Omega_{0}\right)$ into a functional equation satisfied by the multivariate generating function $F(x ; u, v)$ of 0 -skinny slicings, where $x$ takes into account the size of the slicing, while the catalytic variables $u$ and $v$ correspond to the labels $h$ and $k$ of the object. And, with the help of Maple, we compute $F(x ; 1,1)$ using the (usual) kernel method, applied twice.

We point out that D. Callan indicates in [14] that $F$ is also the generating function of Schröder paths with no triple descents, i.e. having no occurrences of the factor $D D D$, where $D$ encodes the down step. It would be interesting to provide a bijection between Schröder slicings and Schröder paths whose restriction to 0-skinny slicings yields a bijection with Schröder paths having no triple descents.

For any $m$, we can apply the same method as in the proof of Theorem 16. From the succession rule $\left(\Omega_{m}\right)$, we therefore obtain a system of $m$ functional equations satisfied by generating functions 
$F_{1}, \ldots, F_{m}$ such that the generating function of $m$-skinny slicings is $F_{1}+\ldots+F_{m}$. We have been able to solve this system in the case $m=2$, using again the kernel method. We obtain an algebraic generating function for 2-skinny slicings, which is however implicit, involving the root of a polynomial of degree 5 . Note that the corresponding enumeration sequence is unknown from [14].

We leave open the question of solving these systems for some $m \geq 3$ or for general $m$, or of deriving from them some information on the nature of the generating function of $m$-skinny slicings. We suspect that these generating functions are algebraic (although letting $m$ go to infinity, we recover the generating function for Baxter numbers which is $D$-finite but not algebraic). We believe this could be proved with an extension of the kernel method to systems of equations for generating functions with catalytic variables.

\subsection{Row-restricted slicings}

Conditions $\left(\ell r_{m}\right)$ naturally generalize the condition that defines Schröder slicings, but it is not the most natural restriction on horizontal blocks of Baxter slicings one may think of. Indeed, for some parameter $m \geq 1$, we could simply impose that horizontal blocks have width no larger than $m$. In what follows, we study these objects under the name of $m$-row-restricted slicings.

Note that, taking $m=1$, we recover Catalan slicings, and that the case $m=0$ is degenerate, since there is only one 0-row-restricted slicing of any given size: the horizontal bar of height 1 and width $n$ divided in (vertical) blocks made of one cell only.

Theorem 17 A generating tree for m-row-restricted slicings is described by the succession rule:

$$
\text { root labeled }(1,1) \quad \text { and } \quad(h, k) \rightsquigarrow\left\{\begin{array}{ll}
(1, k+1),(2, k+1), \ldots,(h, k+1), & \\
(h+1,1),(h+1,2), \ldots,(h+1, k), & \text { if } h<m \\
(m, 1),(m, 2), \ldots,(m, k) . & \text { if } h=m
\end{array} \quad\left(\Upsilon_{m}\right)\right.
$$

Proof: Again, the proof is similar to those of Theorem 3 and 15 , and when a slicing has label $(h, k), h$ (resp. $k$ ) indicates the maximal width of a horizontal block that may be added (resp. the maximal height of a vertical block that may be added). In the case of $m$-row-restricted slicings, when a vertical block is added to the right, the maximal width of a horizontal block that may be added afterward increases by 1 , except if it was $m$ already, in which case it stays at $m$.

Like in the case of $m$-skinny slicings, the succession rule $\left(\Upsilon_{m}\right)$ yields a system of functional equations satisfied by the generating function of $m$-row-restricted slicings. Using again the kernel method (and Maple), we have solved this system for $m=2$, and observed that the generating function of 2-rowrestricted slicings satisfies the equation of Theorem 16. Consequently, although we have no bijective proof for the moment, it holds that:

Theorem 18 The number of 2-row-restricted slicings is equal to the number of 0-skinny slicings, for any fixed size.

Note that it does not hold in general that there are as many $m$-skinny slicings as $m+2$-row-restricted slicings: already for $m=1$, there are 913 -row-restricted slicings but 90 Schröder (i.e., 1-skinny) slicings of size 5 . More precisely, out of the 92 Baxter slicings of size 5 , only $\square \square$ is not 3-row-restricted, but both and $\square$ are not Schröder slicings. 
When it comes to $m$-row-restricted slicings for general $m$, the same questions as for $m$-skinny slicings can be asked: can their generating functions be computed from the system of functional equations derived from rule $\left(\Upsilon_{m}\right)$ ? or can the nature of these generating functions be derived?

\subsection{Other extensions}

We believe our results and the questions left open demonstrate that slicings of parallelogram polyominoes have a rich combinatorics yet to explore. We hope to contribute to this study in future work. We also ask whether similar interesting phenomena may happen when "slicing" other families of polyominoes.

\section{References}

[1] C. Banderier, M. Bousquet-Mélou, A. Denise, P. Flajolet, D. Gardy, D. Gouyou-Beauchamps, Generating functions for generating trees, Disc. Math., vol. 246, pp. 29-55, 2002.

[2] E. Barcucci, A. Del Lungo, E. Pergola, R. Pinzani, ECO: a methodology for the Enumeration of Combinatorial Objects, J. Diff. Eq. and App., vol. 5, pp. 435-490, 1999.

[3] N. Bonichon, M. Bousquet-Mélou, É. Fusy, Baxter permutations and plane bipolar orientations, Séminaire Lotharingien de Combinatoire 61A, article [B61Ah], 2008.

[4] M. Bousquet-Mélou, Four classes of pattern-avoiding permutations under one roof: generating trees with two labels, Electronic J. Combinatorics, vol. 9(2), paper R19, 2003.

[5] M. Bouvel, V. Guerrini, S. Rinaldi, Slicings of parallelogram polyominoes, or how Baxter and Schröder can be reconciled, Preprint available at http://arxiv.org/abs/1511.04864

[6] M. Bouvel, O. Guibert, Refined enumeration of permutations sorted with two stacks and a $D_{8-}$ symmetry, Annals of Combinatorics, vol. 18(2), pp. 199-232, 2014.

[7] S. Burrill, J. Courtiel, É. Fusy, S. Melczer, M. Mishna, Tableau sequences, open diagrams, and Baxter families Preprint available at http://arxiv.org/abs/1506.03544

[8] S. Burrill, S. Elizalde, M. Mishna, L. Yen, A generating tree approach to k-nonnesting partitions and permutations, To appear in Annals of Combinatorics.

[9] F.R.K. Chung, R. Graham, V. Hoggatt, M. Kleiman, The number of Baxter permutations, Journal of Combinatorial Theory Series A, vol.24(3), pp.382-394, 1978.

[10] S. Felsner, É. Fusy, M. Noy, D. Orden, Bijections for Baxter families and related objects, Journal of Combinatorial Theory Series A, vol. 118(3), pp.993-1020, 2011.

[11] S. Gire, Arbres, permutations à motifs exclus et cartes planaires: quelques problèmes algorithmiques et combinatoires, Ph.D. thesis, Université Bordeaux I, 1993.

[12] E. Pergola, R. A. Sulanke, Schröder Triangles, Paths, and Parallelogram Polyominoes, Journal of Integer Sequences, vol. 1, Article 98.1.7, 1998.

[13] L. Shapiro, A. B. Stephens, Bootstrap percolation, the Schröder numbers, and the $N$-kings problem, SIAM Journal on Discrete Mathematics, vol. 4, pp. 275-280, 1991.

[14] OEIS Foundation Inc., The On-line Encyclopedia of Integer Sequences, http://oeis.org, 2011.

[15] J. West, Generating trees and the Catalan and Schröder numbers, Disc. Math., vol. 146, pp. 247$262,1995$. 